\title{
Robótica para la inclusión educativa: una revisión sistemática
}

\author{
Robotics for educational inclusion: a systematic review
}

\author{
Judit Rodrigo Parra \\ Universidad de Murcia (España) \\ judit.rodrigo@um.es
}

Recibido: $16 / 09 / 2021$

Aceptado: $20 / 11 / 2021$

Publicado: 01/12/2021

\section{RESUMEN}

La educación debe ser inclusiva y uno de los instrumentos de los que se puede servir es la robótica. Además, uno de los elementos de la robótica es la programación y para programar se aplica el pensamiento computacional. Por otra parte, para que la educación sea inclusiva se debe atender a la diversidad. Así, se procede a desarrollar una revisión sistemática sobre el uso de la robótica para la inclusión educativa. Las preguntas de investigación son: ¿La robótica educativa puede ser una estrategia de inclusión educativa?, ¿qué beneficios se obtienen con el uso de la robótica en la inclusión educativa? y ¿cómo es la formación de los docentes en relación con el uso de la robótica para la inclusión? Tras aplicar filtros siguiendo la declaración PRISMA han sido seleccionados 23 estudios pertenecientes a las bases de datos Scopus y WOS. Las conclusiones son que la robótica sí puede ser una estrategia de inclusión educativa. Además, se señalan numerosos beneficios de la misma, como el aumento de la motivación, la creatividad y la capacidad de trabajo en equipo y la mejora en habilidades sociales y comunicativas. En lo relativo a la formación docente en este ámbito se han obtenido pocos resultados, pues muy pocos estudios de la revisión sistemática tratan este tema. No obstante, se destaca que la mayoría de los docentes considera la robótica una herramienta útil para la inclusión educativa.

\section{PALABRAS CLAVE}

Educación; robótica; inclusión; formación docente.

\section{ABSTRACT}

Education must be inclusive and one of the tools used to achieve this is robotics. In addition, one of the elements of robotics is programming and computational thinking is applied to program. On the other hand, to ensure that education is inclusive, there must be diversity. I proceed to develop a systematic review on the use of robotics for educational inclusion. The questions of this research are: Can educational robotics be a strategy for educational inclusion? What benefits are obtained with the use of robotics in educational inclusion? How is the training of teachers in relation to the use of robotics for inclusion? After applying filters following the PRISMA statement, 23 studies belonging to the Scopus and WOS databases have been selected. The conclusions are: educational robotics can indeed be a strategy for educational inclusion. In addition, numerous benefits are reported, such as increased motivation, creativity and teamwork skills, and improved social and communication skills. Few results have been obtained about teacher training in this área (very few studies in the systematic review address this issue). However, it is noted that most teachers consider robotics a useful tool for educational inclusion.

\section{KEYWORDS}

Education; robotic; inclusion; teacher training. 


\section{CITA RECOMENDADA}

Rodrigo, J. (2021). Robótica para la inclusión educativa: una revisión sistemática. RIITE Revista Interuniversitaria de Investigación en Tecnología Educativa, 11, 150-171. https://doi.org/10.6018/riite.492211

\section{Principales aportaciones del artículo y futuras líneas de investigación:}

- Principales aportaciones: diferentes usos de la robótica para favorecer la inclusión educativa (telepresencia, etcétera), numerosos beneficios al usar robótica en la inclusión educativa (mejora de habilidades sociales, aumento de motivación e interés por la tarea, etcétera) y una visión acerca de la formación docente en relación a la robótica para la inclusión.

- Futuras líneas de investigación: más estudios que aborden el uso de robótica educativa para la inclusión educativa, pues no parece un tema muy abordado. Por otra parte, realizar otras revisiones sistemáticas con temas concretos de inclusión educativa, como el uso de robótica para la inclusión educativa de alumnado con TEA y su uso con alumnado de altas capacidades o con talentos específicos.

\section{INTRODUCCIÓN}

El tema de estudio es el uso de la robótica para la inclusión educativa en su sentido más amplio (discapacidad, inclusión digital, accesibilidad, inclusión socioeconómica, etcétera), pero siempre ligado al ámbito educativo, incluyendo la formación docente, por considerarla la base fundamental para poder llevar a cabo una robótica educativa que favorezca y beneficie la inclusión educativa.

Los términos "pensamiento computacional" y "programación" suelen encontrarse ligados al de "robótica", ya que la programación es necesaria para el desarrollo de la robótica y, a su vez, se debe aplicar pensamiento computacional para programar, de tal manera que estos tres conceptos están interrelacionados. Por otra parte, la educación debe ser inclusiva, para lo cual se debe atender a la diversidad, por lo que parece razonable centrarse en este aspecto dentro del uso de robótica en el ámbito educativo.

Por todo ello, el objetivo de este estudio es describir el uso de la robótica para la inclusión educativa, sus beneficios y la formación docente al respecto, todo ello en base a los estudios existentes.

\subsection{PENSAMIENTO COMPUTACIONAL}

El pensamiento computacional ha cobrado relevancia en los últimos años, sin embargo, no existe una definición claramente consensuada (Shute et al., 2017). No obstante, la realizada por Wing (2006) ha adquirido una trascendental importancia (Zapata-Ros, 2015; Shute et al., 2017). Wing (2006) define el pensamiento computacional como "un proceso que implica resolver problemas, diseñar sistemas y entender el comportamiento humano, haciendo uso de los conceptos fundamentales de la informática" (p. 33).

Así, Basogain \& Olmedo (2020) defienden el pensamiento computacional como una habilidad para resolver problemas cotidianos con creatividad mediante algoritmos mentales, permitiendo una manera de resolución diferente incluso para problemas complejos (Ángel-Díaz et al., 2020). Siendo, además, la solución resultante eficaz (Avello-Martínez et al., 2020).

Wing (2006) destaca que al hablar de pensamiento computacional lo importante son las ideas y no los dispositivos. De hecho, el pensamiento computacional se puede desarrollar separadamente de los ordenadores y la tecnología (Basogain, \& Olmedo, 2020). Pues, siguiendo a Zapata-Ros (2015), trata sobre la resolución de problemas y otras habilidades como la creatividad, el pensamiento abstracto y un largo etcétera. 
Respecto a la evaluación del pensamiento computacional, podemos decir que a tenor de las diversas definiciones que encontramos para el mismo, no es de extrañar que no exista una manera de evaluarlo ampliamente apoyada (Shute et al., 2017). Así, realizar un instrumento de evaluación para esta habilidad es una tarea compleja, de manera que evaluarlo supone un reto (Avello-Martínez et al., 2020).

El pensamiento computacional se aplica al programar, por lo que va unido a la programación.

\subsection{PROGRAMACIÓN}

El aprendizaje de programación se puede dar desde la infancia. De hecho, existen diversas plataformas, cada vez más, dirigidas a niños de distintas edades con la intención de potenciar el aprendizaje de creación de juegos o actividades mediante una interfaz amigable (Martínez et al., 2018).

A este respecto, podemos destacar Scratch, un entorno de programación por bloques que constituye un método de programación simplificado que permite la creación de proyectos propios. Además, tiene su versión ScratchJr destinada a partir de los cinco años (MirandaPinto, 2019).

La programación es un elemento de la robótica, pues para desarrollar la robótica es necesario programar.

\subsection{ROBÓTICA EDUCATIVA}

Siguiendo a Pittí et al. (2014) podemos describir la robótica educativa "como un proceso sistemático y organizado, en el que intervienen elementos tecnológicos interrelacionados (plataforma robótica y software de programación) como herramientas mediadoras, cuyo objetivo final es lograr aprendizajes" (p. 41).

Gracias a la robótica educativa el alumnado puede participar activamente en el proceso de enseñanza-aprendizaje, dado que construyen por ellos mismos diversos proyectos que les acercan a la vida real. Erigiéndose así la robótica en una herramienta generadora de pensamiento que posibilita el entender la educación de una forma nueva (Quiroga, 2018).

La robótica educativa se fundamenta, como afirman Kucuk \& Sisman (2018), en la teoría constructivista y en la teoría construccionista, considerándose los postulados de Piaget y Papert un hito para este campo. Ambos, aunque con teorías diferentes, convergen en que los estudiantes aprenden mejor cuando forman parte activa del proceso de aprendizaje, algo que la robótica educativa posibilita.

Es importante que las intervenciones de robótica educativa estén debidamente diseñadas para optimizar los resultados (Kucuk, \& Sisman, 2018), pues el simple hecho de realizar actividades de robótica educativa no supone en sí mismo un aprendizaje (Avello-Martínez et al., 2020), ya que este debe estar debidamente planificado. De manera que, para hacer frente a este desafío, es fundamental una buena formación docente en este ámbito que les capacite en su uso en una doble vertiente que aglutine tanto los aspectos didácticos como los técnicos (Pittí et al., 2014), dado que el beneficio o no del uso de robótica depende de su puesta en práctica por parte del docente (Roberts-Yates, \& Silvera-Tawil, 2019).

Tal y como señalan Orcos \& Aris (2019) la robótica educativa está cada vez más presente en las distintas etapas educativas, siendo una de sus características que posibilita un enfoque globalizador de las distintas áreas que componen el currículum.

Así, Sánchez \& Juárez (2017) ponen en valor ciertos beneficios del uso de robots en el proceso educativo (aumento de la creatividad, mejora del trabajo en equipo, etc.) defendiendo, además, que los estudiantes que reciben formación con robótica educativa tienen menor probabilidad de sufrir fracaso escolar. Por otra parte, señalan que el aprendizaje mediante el uso de robots educativos es mejor que cuando se da únicamente a través de software. No obstante, se debe tener en cuenta que también existen simuladores y, aunque estos despierten menos motivación en el alumnado (Ángel-Díaz et al., 2020), son también una opción a valorar que puede ser muy útil y más accesible en términos económicos. 
Hemos visto diversas características y beneficios del pensamiento computacional, la programación y la robótica educativa. Vamos ahora a analizar los conceptos de educación inclusiva y atención a la diversidad.

\subsection{EDUCACIÓN INCLUSIVA}

La educación inclusiva no abarca únicamente al alumnado con dificultades de aprendizaje o discapacidad, si no que implica una transformación de los sistemas educativos para lograr el desarrollo pleno e igualdad de oportunidades de todo el alumnado (Echeita, 2017). Así, una educación inclusiva supone proporcionar a todo el alumnado una educación de calidad (García et al., 2018), sean cuales sean sus características. En este sentido afirma Ainscow (2016) que para que las escuelas sean inclusivas deben atender a todo su alumnado respondiendo a sus características propias, de manera que todos se beneficien.

Internacionalmente se entiende el término educación inclusiva en su significado más amplio, pretendiendo atender a la diversidad en los distintos campos de la vida (Echeita, \& Ainscow, 2011).

Por otra parte, para llevar a cabo una educación inclusiva efectiva se debe realizar una evaluación de las tareas realizadas en este sentido (García et al., 2018), pues esto ayuda al docente a mejorar al respecto.

En este marco de inclusión, la tecnología forma un papel importantísimo, erigiéndose como un elemento muy útil para compensar desigualdades o desventajas de los alumnos. Para que esto sea posible es necesario una formación por parte de los docentes que les capacite para ello, de hecho, las carreras universitarias inciden cada vez más tanto en la instrucción referida a la inclusión, como la referente a la tecnología (Maestre et al., 2017).

Tal y como señalan Moriña \& Carballo (2018) la predisposición de los docentes a recibir una mayor formación que les permita desarrollar una educación inclusiva con su alumnado con discapacidad.

Como se puede observar, parece fundamental, incluso ineludible, hablar de atención a la diversidad al estar hablando de educación inclusiva.

\subsection{ATENCIÓN A LA DIVERSIDAD}

La atención a la diversidad se encuentra en continua evolución, pues la sociedad es cambiante y, por ende, sus demandas también, debiendo los centros educativos tomar las medidas oportunas para que el alumnado logre desarrollar al máximo sus capacidades y pueda participar favorablemente en la sociedad. Todo ello enmarcado en un sistema educativo que también vele por dicho propósito (Miranda et al., 2018).

Conviene señalar que, tal y como apunta Azorín (2017), la equidad se ha visto perjudicada por la competición creada en las escuelas, pues los instrumentos para medir el éxito del alumnado pueden ser excluyentes. Siendo fundamental revisar estos criterios y realizarlos teniendo en cuenta que se debe lograr la atención plena a todos los estudiantes independientemente de sus características (Ainscow, 2016).

Añade Azorín (2017) como hecho positivo el que hayan surgido numerosos instrumentos para medir la atención a la diversidad que se ofrece y el grado de inclusión logrado, pero señala que quiénes los aplican suelen pertenecer a la comunidad científica en lugar de a la escolar, lo cual puede suponer un escollo y, apunta, que lo deseable sería la segunda opción.

Los centros y los docentes deben adaptarse para poder dar una respuesta educativa que atienda a la diversidad y servirse de las estrategias que tiene a su alcance.

\section{MÉTODO}

Dada la necesidad e importancia de lograr una inclusión educativa efectiva y el valor creciente de la robótica educativa, se plantea aquí una revisión sistemática para dar respuesta a una serie de preguntas al respecto de la unión de ambos temas. De manera que el objetivo 
de este estudio es describir el uso de la robótica para la inclusión educativa, sus beneficios y la formación docente al respecto, todo ello en base a los estudios existentes.

Por otra parte, para llevar a cabo la presente revisión sistemática se han tenido en cuenta los ítems de la declaración PRISMA (Urrútia, \& Bonfill, 2010) y la lista de verificación de CASP (Critical Appraisal Skills Programme, 2018).

\subsection{PREGUNTAS DE INVESTIGACIÓN}

La presente investigación pretende responder a las siguientes preguntas:

1. ¿La robótica educativa puede ser una estrategia de inclusión educativa?

2. ¿Qué beneficios se obtienen con el uso de la robótica en la inclusión educativa?

3. ¿Cómo es la formación de los docentes en relación con el uso de la robótica para la inclusión?

\subsection{ESTRATEGIAS DE BÚSQUEDA}

Para realizar el proceso de búsqueda se han utilizado dos bases de datos: Scopus y WOS (Web of Science).

Se ha realizado solamente una búsqueda decidida tras diversas pruebas. Así, los conceptos buscados han sido: "Robotic* and inclusi* and (education* or learn* or student*)". De manera que la cadena de búsqueda ha sido: TITLE-ABS-KEY (robotic* AND inclusi* AND ( education* OR learn* OR student*)) AND ( LIMIT-TO (LANGUAGE, "English") OR LIMIT-TO ( LANGUAGE, "Spanish" ) ).

\subsection{CRITERIOS DE SELECCIÓN}

Los criterios de inclusión han sido:

- Estudios en español o inglés.

- Todo tipo de documentos (artículos, conferencias, etcétera).

- Investigaciones que respondan a las preguntas de esta revisión sistemática.

Partiendo de los resultados de la búsqueda en ambas bases de datos, se ha realizado la primera revisión leyendo títulos, resúmenes y palabras claves para seleccionar solamente los que sirven y excluir el resto. Posteriormente, se ha llevado a cabo una segunda revisión mediante la lectura completa de todos los estudios incluidos en la fase anterior para determinar los que finalmente son válidos y seleccionables. revisión.

La tabla 1 muestra los resultados de la búsqueda y los estudios incluidos tras cada

Tabla 1. Resultados de la búsqueda y revisiones

\begin{tabular}{cccc}
\hline Base de datos & $\begin{array}{c}\text { Resultado de la } \\
\text { búsqueda }\end{array}$ & Primera revisión & Segunda revisión \\
\hline Scopus & 420 & 125 & 22 \\
WOS & 267 & 24 & 1 \\
Total & 687 & 149 & 23 \\
\hline
\end{tabular}

\subsection{EXTRACCIÓN DE DATOS}

Tras la búsqueda en ambas bases de datos se obtienen 687 documentos, 420 de Scopus y 267 de WOS.

Tras la primera revisión, basada en la lectura de los títulos, resúmenes y palabras claves, quedan 125 investigaciones de Scopus y 24 de WOS, o sea 149 en total. Por último, tras la exclusión de los 26 estudios no encontrados en texto completo (17 de Scopus y 9 de WOS), la lectura completa de los 123 incluidos hasta este momento y la aplicación de los criterios de 
inclusión quedan 22 documentos de Scopus y 1 de WOS, lo que suman, 23 documentos en total seleccionados.

Los estudios duplicados aparecen descontados en la primera revisión y han sido contabilizados una sola vez. Los estudios presentes en ambas bases de datos han sido contabilizados en Scopus y excluidos en WOS, por eso la cifra de documentos cogidos de WOS es muy inferior a la resultante en Scopus.

A continuación, se muestra en la figura 1 el proceso de búsqueda que se ha relatado.

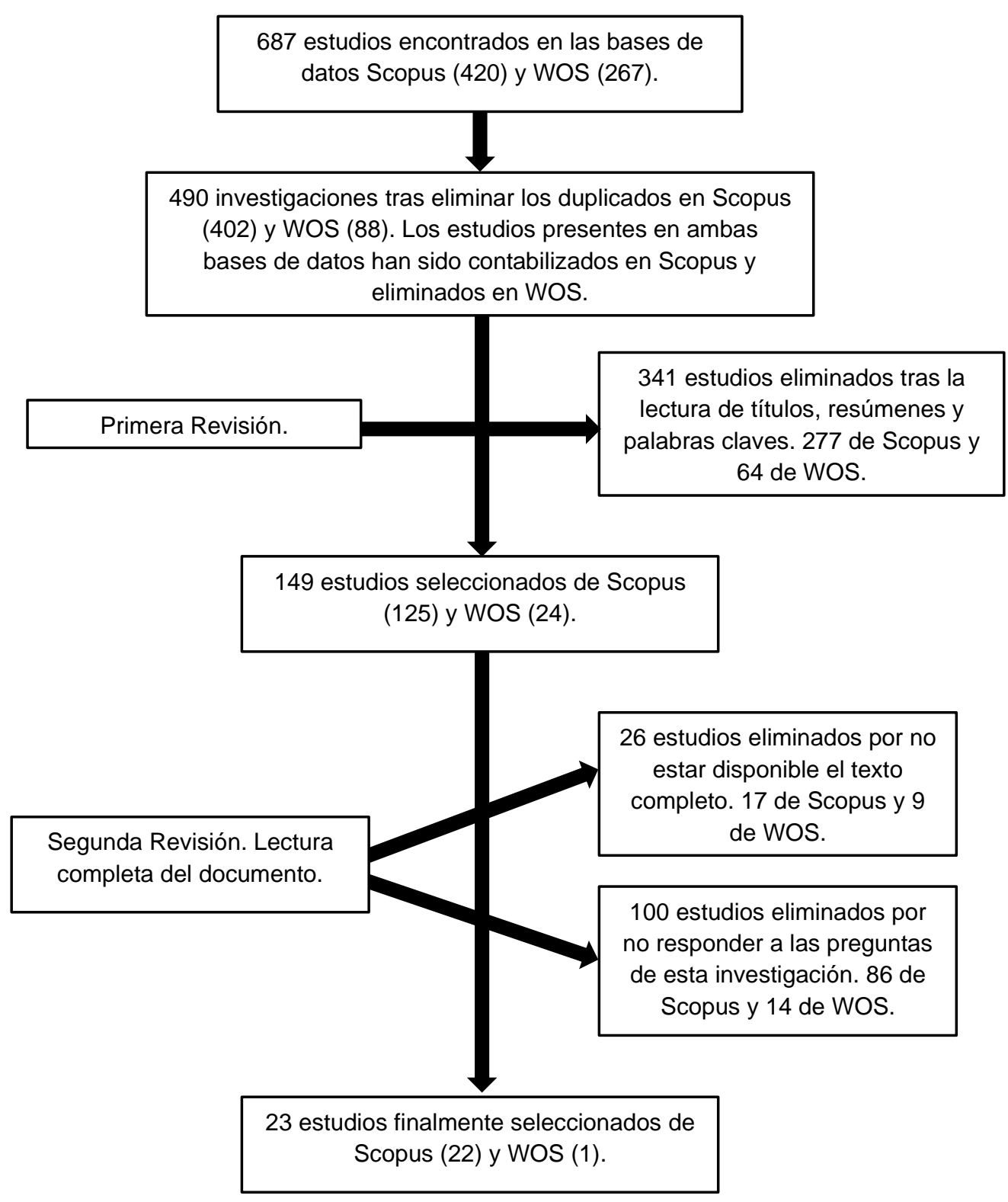

Figura 1. Proceso de búsqueda y selección de documentos 


\section{RESULTADOS}

\subsection{RESULTADOS DE LA BÚSQUEDA}

Tras el proceso de búsqueda han sido seleccionados 23 documentos, los cuales se reflejan en la tabla 2 especificando de cada uno de ellos los siguientes datos: título, autor/a/es/as, año de publicación, país, palabras claves, número de participantes o estudios (según el tipo de investigación, por ejemplo, en caso de la revisión sistemática se señalan los estudios, mientras que si se trata de un estudio de caso se indican los participantes), tipo de documento, metodología y conclusiones.

La mayoría de documentos resultantes datan del año 2015 hacia el presente, a excepción de dos documentos del año 2006. Por otra parte, 16 de los 23 documentos son artículos y 7 son conferencias en papel.

\subsection{EVALUACIÓN DE LA CALIDAD DE LOS ESTUDIOS}

La calidad de los estudios ha sido evaluada realizando una adaptación de la lista de control para revisiones sistemáticas de CASP (Critical Appraisal Skills Programme, 2018).

La finalidad de dicha evaluación es comprobar la calidad y fiabilidad de los estudios y determinar si ésta es suficiente para quedar incluidos en la revisión sistemática. En este caso ningún estudio ha sido excluido tras la evaluación.

A continuación, se muestra en la tabla 3 el resultado para cada pregunta en cada estudio. También se incluye el dato del total de respuestas "sí", "no" y "no sé" que ha recibido cada estudio. 
Tabla 2. Descripción de los estudios

\begin{tabular}{|c|c|c|c|c|c|c|}
\hline Título, autor/a/es/as y año & País & Palabras claves & $\begin{array}{c}\text { № de } \\
\text { participantes o } \\
\text { estudios }\end{array}$ & $\begin{array}{c}\text { Tipo de } \\
\text { documento }\end{array}$ & Metodología & Conclusiones \\
\hline $\begin{array}{l}\text { 1. A case study of a robot- } \\
\text { assisted speech therapy for } \\
\text { children with language } \\
\text { disorders. Estévez et al. } \\
\text { (2021). }\end{array}$ & España. & $\begin{array}{l}\text { NAO robot, social robot, } \\
\text { speech therapy, inclusive } \\
\text { education and children with } \\
\text { language disorders. }\end{array}$ & 5 & Artículo. & Estudio de caso. & $\begin{array}{l}\text { Refleja el potencial de usar un } \\
\text { robot NAO en terapia del habla } \\
\text { y consecución de objetivos } \\
\text { educativos de alumnado (entre } \\
9 \text { y } 12 \text { años) con discapacidad. }\end{array}$ \\
\hline $\begin{array}{l}\text { 2. Robótica educativa en } \\
\text { contextos inclusivos: el } \\
\text { caso de las aulas } \\
\text { hospitalarias. González- } \\
\text { González et al. (2021). }\end{array}$ & España. & $\begin{array}{l}\text { Emoción, pensamiento } \\
\text { computacional, escuela en } \\
\text { hospitales, pedagogía } \\
\text { diferencial y pedagogía } \\
\text { hospitalaria. }\end{array}$ & 22 & Artículo. & Estudio de caso. & $\begin{array}{l}\text { El alumnado del aula } \\
\text { hospitalaria (entre } 4 \text { y } 16 \text { años } \\
\text { en este estudio) aprende } \\
\text { pensamiento computacional } \\
\text { mediante robótica y, además, } \\
\text { sus emociones mejoran. }\end{array}$ \\
\hline $\begin{array}{l}\text { 3. Assessing the impact of } \\
\text { an adapted robotics } \\
\text { programme on interest in } \\
\text { Science, Technology, } \\
\text { Engineering and } \\
\text { Mathematics (STEM) } \\
\text { among children with } \\
\text { disabilities. Lamptey et al. } \\
\text { (2019). }\end{array}$ & Canadá. & $\begin{array}{l}\text { Children with disabilities, } \\
\text { computing, inclusion, } \\
\text { inclusive STEM, LEGO, } \\
\text { programming, robotics and } \\
\text { youth. }\end{array}$ & 57 & Artículo. & Estudio de caso. & $\begin{array}{l}\text { Señala que mediante robótica } \\
\text { adaptada se logra enseñar } \\
\text { informática y robótica a niños } \\
\text { con diversas discapacidades y } \\
\text { que, también, fomenta el } \\
\text { desarrollo de otras habilidades. }\end{array}$ \\
\hline $\begin{array}{l}\text { 4. Back to school with } \\
\text { telepresence robot } \\
\text { technology: a qualitative } \\
\text { pilot study about how } \\
\text { telepresence robots help } \\
\text { school-aged children and } \\
\text { adolescents with cancer to } \\
\text { remain socially and } \\
\text { academically connected } \\
\text { with their school classes } \\
\text { during treatment. Weibel et } \\
\text { al. }(2020) \text {. }\end{array}$ & Dinamarca. & $\begin{array}{l}\text { Cancer, childhood illness, } \\
\text { education, school nursing } \\
\text { and technology. }\end{array}$ & 27 & Artículo. & $\begin{array}{l}\text { Estudio piloto } \\
\text { cualitativo. }\end{array}$ & $\begin{array}{l}\text { Robot de telepresencia como } \\
\text { elemento inclusivo para } \\
\text { estudiantes con cáncer, pues } \\
\text { les permite estar conectados } \\
\text { social y académicamente con su } \\
\text { clase. }\end{array}$ \\
\hline $\begin{array}{l}\text { 5. Coding through touch: } \\
\text { exploring and re-designing }\end{array}$ & Estados Unidos. & $N / A$ & 9 & $\begin{array}{l}\text { Conferencia en } \\
\text { papel. }\end{array}$ & Estudio de caso. & $\begin{array}{l}\text { Analiza beneficios y dificultades } \\
\text { de emplear un kit robótico de }\end{array}$ \\
\hline
\end{tabular}


tactile making activities with learners with visual

dis/abilities. Seo y Richard (2020).

\section{WebMoti. Asmy et al.} (2020).

Canadá.

Video conferencing robotics, Autism Spectrum Disorder and inclusive design.

\section{Autism Spectrum Disorders,} social robotics, special educational needs and assistive technology.

Spectrum Disorders in

mainstream classrooms: an overview of the RoBò

service delivery approach.

Desideri et al. (2020).

\section{Children with disabilities} engaging in STEM:

exploring how a group-

based robotics program

influences STEM activation. Lindsay et al. (2019).

\section{Educational robotics in} Down syndrome: a feasibility study. Bargagna et al. (2018).

10. Developing a protocol using a humanoid robot to assist children with autism

robotics, under-represented

Reino Unido. Assistive robotics, autism, codificación táctil con alumnado con discapacidad visual. Señala su facilidad de manipulación, pero con algún problema en su uso de manera independiente por el alumnado.

Expone que alumnado con TEA gracias al uso de WebMoti se mantiene conectado con su clase cuando no pueden acudir presencialmente. Observan beneficios educativos y algunas dificultades técnicas.

Muestra una visión general de uso del sistema RoBò para emplear robots sociales que aumenten las oportunidades de interacción social y de inclusión de niños con TEA. La

conclusión es favorable.
Canadá.

Disability, inclusion,

groups, autism and physical disability.

Educational robotics, executive functions, Down syndrome and Bee-Bot.
Artículo

Estudio de caso.

Refleja que incluir a estudiantes con discapacidad (muestra de 6 a 14 años) en un programa de robótica ayuda a desarrollar sus habilidades en STEM.

\section{Italia.}

Artículo.

Estudio de caso
Determina que el robot Bee-Bot es útil para fomentar el interés, atención e interacción de alumnado con síndrome de Down. Sin embargo, concluye no poder afirmar que desarrolle las funciones ejecutivas, aunque sí mejora alguna habilidad.

Artículo.

Estudio de caso.
Indica que mediante una robótica lúdica se pueden potenciar las habilidades de toma de perspectiva visual 
to develop visual

perspective taking skills.

Wood et al. (2019)

11. Better education

opportunities for students

with autism and intelectual

disabilities through digital

technology. Roberts-Yates

y Silvera-Tawil (2019).

12. A study to design $\mathrm{V}$ classrooms using virtual

reality aided telepresence.

Jadhav et al. (2018).

13. Robotic teaching aid for disabled children: a

sustainable solution for

Industrial Revolution 4.0

Zamin et al. (2018).

\section{Adapting a robotics} program to enhance

participation and interest in

STEM among children with

disabilities: a pilot study.

Lindsay y Hounsell (2016).

15. Using assistive robots to promote inclusive

education. Encarnação et

al. (2016).

16. A training course in educational robotics for
Equal education, autism

intelectual disability,

technology, social robotics

India.

Telepresence, virtual

reality, robotics, simulators,

virtual inclusion and bot.

Malasia.

Autism, developmental

disabilities, Down

syndrome, robotic, slow

learner, social interaction

and special education.

Canadá.

LEGO robotics, play,

inclusion, children, physica disability, autism, inclusive

STEM, underserved youth,

Elementary school and

$$
\text { robotics. }
$$

Assistive robots,

augmentative manipulation

and inclusive education. and virtual reality.

Conferencia en

papel.

$\mathrm{N} / \mathrm{A}$

Artículo.

Estudio de caso

Estudio empírico cualitativo.

Estudio de caso.

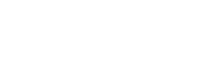

studio piloto de métodos mixtos: encuestas,

observaciones y entrevistas previas y posteriores al taller.

Investigación

descriptiva de un estudio de caso.

Educational robotics, learning support teacher (capacidad de ver el mundo desde el lugar de otra persona) de los niños con TEA.

Muestra los robots de asistencia social como una herramienta que aumenta la interacción, entre otros aspectos sociales y educativos, de alumnado con autismo y discapacidad intelectual.

Concluye que usando realidad virtual con robots de

telepresencia se pueden crear aulas inclusivas para alumnado con discapacidad.

Sostiene que el uso de robótica en educación especial mejora el aprendizaje de estos niños y potencia su inclusión. El robot realiza movimientos repetitivos por los cuales estos niños se sienten atraídos y aprenden.

Concluye que se debe involucrar a los jóvenes en robótica para mejorar su interés y habilidades en STEM.

Exponen que el uso de robots de asistencia logra una participación más inclusiva del alumnado con discapacidad, aunque plantea algunos problemas (necesidad de otro docente y de más tiempo).

La mayoría de docentes valoran positivamente la utilización de 
learning support teachers.

Agatolio et al. (2017)

\section{R2T2: Robotics to} integrate educational

efforts in South Africa and

Europe Mondada et al.

(2016).

18. Analysis of the use of a robot to improve social

skills in children with autism

spectrum disorder. Valadão et al. (2016)

\section{Robots \& NEE}

Learning by playing with

robots in an inclusive

school setting. Conchinha y

Correia de Freitas (2015).

20. Playful leaming:

Educational robotics

applied to students with

learning disabilities.

Conchinha et al. (2015).

21. Technology in Finnish special education. Toward inclusion and harmonized school days. Kärnä-Lin et al. (2006). special needs education,

inclusive education, teacher

training and course

evaluation.

\section{Suiza y}

Sudáfrica.

Rescue, educational

robotics, Thymio robot,

space robotics y

programming.

Brasil y

Argentina.

Autism spectrum disorder, social skills, social robots and assistive technology.

Portugal.

Educational robotic, Lego Mindstorms, inclusive

School and Play in

education.

Portugal y

Brasil.

Educational robotics, Lego

Mindstorms, learning

disabilities, inclusion and playful learning.

Finlandia

Technologies of special education, educational

robotics, inclusive

education and Finnish

special education. robótica educativa,

concretamente manifiestan

estar a favor de su uso con

estudiantes con TEA, TDAH,

etcétera.

Concluye que el trabajo colaborativo de robótica entre

alumnado de Sudáfrica y

Europa muestra amplios

beneficios Observándose

mejora en los participantes de

Sudáfrica, ya que se benefician

de trabajar con estudiantes con

mayor práctica en este tema.

Determina que utilizar un robot

para estimular las habilidades

sociales de niños con TEA es

eficaz, puesto que se logró

potenciar en 4 de 5 niños con

TEA.

26 estudiantes y Conferencia en Estudio empírico.

Tras los talleres todos los

docentes respondieron que la

robótica educativa promueve la

inclusión y que la potencia más que otras actividades.

Concluye que a través de la

robótica educativa los alumnos mejoran sus interacciones sociales y también conceptos teóricos útiles.

Analiza el uso de tecnología (incluida robótica) en Finlandia con alumnado de educación

especial y concluye que su uso es escaso. Afirma que aprender a programar con robótica mejora las habilidades sociales del alumnado de educación 
22. RoboEduc: A software for teaching robotics to

technological excluded

children using $L E G O$

prototypes. Barrios-

Aranibar et al. (2006)

23. Educational intervention España.

for students with ASD:

emotion production and

recognition through the

Bee-Bot robot. Pérez et al.

(2019).

\section{Brasil.}

(n)

\section{Digital inclusion,}

educational software, robot

programming, LEGO robot

y educational robotics.

Robots, perceptions,

emotional management and

autism spectrum disorder.
24 niños/as. 12

de tercer grado

y 12 de cuarto

grado. De los

cuales 12 son

niñas y 12 son

niños. Divididos

en cuatro

grupos.

2
Conferencia en Estudio de caso.

papel.

Conferencia en Estudio de caso.

papel.

\section{Estudio}

metodológico

cuantitativo

descriptivo. especial.

Exponen que los robots son

mejor acogidos que solamente

los ordenadores y que LEGO

Mindstorms es una buena

opción para favorecer la

inclusión digital.

Concluye que la percepción sobre las emociones de los estudiantes por parte de los docentes es diferente a la de los investigadores en dos de las

tres preguntas de investigación. Los autores emplazan a realizar un estudio más amplio y

solventar limitaciones para

poder tener una conclusión más certera. 
Tabla 3. Evaluación de la calidad de los estudios en base a la lista de verificación de CASP

\begin{tabular}{|c|c|c|c|c|c|c|c|c|c|c|c|c|c|c|c|c|c|c|c|c|c|c|c|}
\hline $\begin{array}{c}\text { Lista de } \\
\text { verificación } \\
\text { de CASP }\end{array}$ & 1 & 2 & 3 & 4 & 5 & 6 & 7 & 8 & 9 & 10 & 11 & 12 & 13 & 14 & 15 & 16 & 17 & 18 & 19 & 20 & 21 & 22 & 23 \\
\hline $\begin{array}{l}\text { ¿El tema de } \\
\text { investigación } \\
\text { está } \\
\text { claramente } \\
\text { definido? }\end{array}$ & Sí & Sí & Sí & Sí & Sí & Sí & Sí & Sí & Sí & Sí & Sí & Sí & Sí & Sí & Sí & Sí & Sí & Sí & Sí & Sí & Sí & Sí & Sí \\
\hline $\begin{array}{l}\text { ¿Los autores } \\
\text { buscaron } \\
\text { correctamente } \\
\text { los } \\
\text { documentos? }\end{array}$ & Sí & Sí & Sí & Sí & $\begin{array}{l}\text { No } \\
\text { sé }\end{array}$ & Sí & Sí & Sí & Sí & Sí & Sí & Sí & Sí & Sí & Sí & Sí & Sí & Sí & Sí & Sí & Sí & $\mathrm{Si}$ & Sí \\
\hline $\begin{array}{l}\text { ¿Cree que se } \\
\text { incluyeron los } \\
\text { estudios } \\
\text { importantes y } \\
\text { pertinentes? }\end{array}$ & Sí & Sí & Sí & Sí & No & No & $\begin{array}{l}\text { No } \\
\text { sé }\end{array}$ & Sí & $\begin{array}{l}\text { No } \\
\text { sé }\end{array}$ & Sí & Sí & No & No & Sí & Sí & Sí & No & Sí & Sí & $\begin{array}{l}\text { No } \\
\text { sé }\end{array}$ & No & Sí & $\begin{array}{l}\text { No } \\
\text { sé }\end{array}$ \\
\hline $\begin{array}{l}\text { ¿Los autores } \\
\text { han tenido en } \\
\text { cuenta todos } \\
\text { los factores } \\
\text { importantes } \\
\text { de confusión? }\end{array}$ & Sí & Sí & Sí & Sí & Sí & Sí & Sí & Sí & Sí & Sí & Sí & $\begin{array}{l}\text { No } \\
\text { sé }\end{array}$ & $\begin{array}{l}\text { No } \\
\text { sé }\end{array}$ & Sí & Sí & Sí & Sí & Sí & Sí & No & Sí & Sí & $\begin{array}{l}\text { No } \\
\text { sé }\end{array}$ \\
\hline $\begin{array}{l}\text { ¿Los } \\
\text { resultados } \\
\text { están } \\
\text { conectados } \\
\text { con los } \\
\text { objetivos? }\end{array}$ & Sí & Sí & Sí & Sí & Sí & Sí & Sí & Sí & Sí & Sí & Sí & $\begin{array}{l}\text { No } \\
\text { sé }\end{array}$ & Sí & Sí & Sí & Sí & Sí & Sí & Sí & Sí & Sí & Sí & Sí \\
\hline $\begin{array}{l}\text { ¿Los } \\
\text { resultados son } \\
\text { precisos? }\end{array}$ & Sí & Sí & Sí & Sí & Sí & Sí & Sí & Sí & $\begin{array}{l}\text { No } \\
\text { sé }\end{array}$ & Sí & Sí & No & Sí & Sí & $\begin{array}{l}\text { No } \\
\text { sé }\end{array}$ & Sí & Sí & Sí & Sí & Sí & Sí & Sí & $\begin{array}{l}\text { No } \\
\text { sé }\end{array}$ \\
\hline $\begin{array}{l}\text { ¿Cree en los } \\
\text { resultados? }\end{array}$ & Sí & Sí & Sí & Sí & Sí & Sí & Sí & Sí & Sí & Sí & Sí & $\begin{array}{l}\text { No } \\
\text { sé }\end{array}$ & Sí & Sí & Sí & Sí & Sí & Sí & Sí & Sí & Sí & Sí & $\begin{array}{l}\text { No } \\
\text { sé }\end{array}$ \\
\hline
\end{tabular}




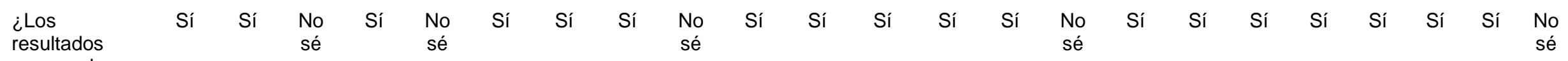

con otros

disponibles?

¿Los Sí Sí Sí Sí Sí Sí Sí Sí Sí Sí Sí Sí Sí Sí Sí Sí Sí Sí Sí Sí No Sí Sí

resultados son

otros

contextos?

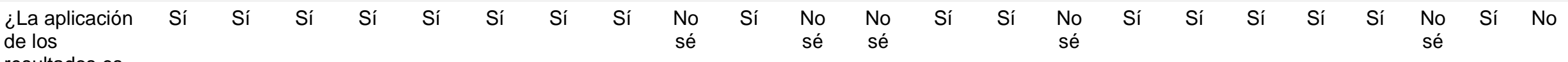

resultados es

rentable en

relación a sus

beneficios y

perjuicios?

\begin{tabular}{lccccccccccccccccccccccccc} 
Total Sí & 10 & 10 & 9 & 10 & 7 & 9 & 9 & 10 & 6 & 10 & 9 & 4 & 8 & 10 & 7 & 10 & 9 & 10 & 10 & 8 & 7 & 10 & 4 \\
Total No & 0 & 0 & 0 & 0 & 1 & 1 & 0 & 0 & 0 & 0 & 0 & 2 & 1 & 0 & 0 & 0 & 1 & 0 & 0 & 1 & 2 & 0 & 1 \\
Total No sé & 0 & 0 & 1 & 0 & 2 & 0 & 1 & 0 & 4 & 0 & 1 & 4 & 1 & 0 & 3 & 0 & 0 & 0 & 0 & 1 & 1 & 0 & 5 \\
\hline
\end{tabular}




\section{DISCUSIÓN Y CONCLUSIONES}

\section{1 ¿LA ROBÓTICA EDUCATIVA PUEDE SER UNA ESTRATEGIA DE INCLUSIÓN EDUCATIVA?}

La robótica educativa como estrategia de inclusión educativa se usa en diversos enfoques como vamos a ver a continuación.

Weibel et al. (2020) y Jadhav et al. (2018) muestran como alumnos que no pueden asistir físicamente a sus aulas, por diversos motivos, gracias al uso de robótica de telepresencia pueden estar presentes en ellas. Señalando Weibel et al. (2020) la importancia de ubicar en el lugar correcto del aula el robot. Fitter et al. (2018) van más allá al realizar un estudio sobre los efectos de personalizar el robot de telepresencia para la educación, sobre lo cual concluyen que al alumnado les gusta.

Acerca de la importancia o no de la apariencia del robot, Roberts-Yates \& Silvera-Tawil (2019) y Valadão et al. (2016) destacan que sí es importante para lograr tener éxito en la tarea, señalando Valadão et al. (2016) que el alumnado se siente más cómodo cuando los robots son de su misma altura. Sin embargo, Bae \& Han (2017) no visualizan ventajas al usar un robot de tamaño real, respecto a uno más pequeño. Aunque es importante remarcar el hecho de que esta investigación no trata el tema de la inclusión, por lo que la disparidad de resultados entre estudios puede deberse a este hecho. Por tanto, la apariencia del robot, incluyendo su altura, sí puede ser importante e influir en el éxito de la actividad, sobre todo cuando se trata de alumnado de educación especial.

Otro aspecto que conviene remarcar en el uso de robótica de telepresencia es el problema con la conexión WIFI indicado por numerosos autores, como Weibel et al. (2020), Mondada et al. (2016), Asmy et al. (2020) y Fitter et al. (2018). Vemos, pues, que es un escollo a tener en cuenta. Para resolver esto, por ejemplo, se pueden poner amplificadores de la señal WIFI en el centro para que más espacio esté cubierto por la misma.

Por otra parte, Zamin et al. (2018), Encarnação et al. (2016), Estévez et al. (2021), Conchinha \& Correia de Freitas (2015), Conchinha et al. (2015), Lamptey et al. (2019), Lindsay \& Hounsell (2016) y Lindsay et al. (2019) muestran que gracias al uso de robótica se logra la inclusión educativa de alumnado con diversas discapacidades o dificultades de aprendizaje, facilitándoles la adquisición de conocimientos en diversos sentidos. Misma idea que apostilla Estévez et al. (2021), al afirmar que su uso en las escuelas promueve una educación más inclusiva.

Dentro de las estrategias de inclusión educativa también se encuentra la adaptación en sí de la herramienta de robótica. Así, vemos el uso de bloques táctiles como parte del kit de robótica para su manejo por parte de alumnado con diversos tipos de discapacidad visual (Seo \& Richard, 2020). También la utilización de un controlador de robot con botones de gran tamaño fáciles de presionar y una aplicación con posibilidad de ajustar el color y otros aspectos visuales (Jadhav et al., 2018). También Zhang \& Hansen (2020) presentan un estudio sobre el manejo de robots de telepresencia mediante la mirada por parte de personas con discapacidad motórica. No obstante, conviene señalar que el diseño accesible no siempre es universal (Seo \& Richard, 2020).

Otro terreno en el que se observa el uso de robótica como estrategia de inclusión educativa es con alumnado con Trastorno del Espectro Autista (TEA), siendo este un colectivo bastante trabajado en diversos estudios (Asmy et al., 2020; Desideri et al., 2020; Pérez et al., 2019).

A este respecto, autores como Roberts-Yates \& Silvera-Tawil (2019) y Valadão et al. (2016) concluyen que las habilidades sociales de alumnado con TEA mejoran con el uso de robots sociales, lo cual favorece su inclusión educativa e, incluso, como señalan Roberts-Yates \& Silvera-Tawil (2019), se logran mejoras al respecto de estas habilidades que con otras tecnologías no es posible. 
Por tanto, se observa, tras analizar diversos estudios, diferentes maneras en las que el uso de robótica se erige como una estrategia de inclusión educativa.

El uso más obvio de la robótica como estrategia de inclusión educativa ha sido para conseguir la telepresencia de alumnado que no puede asistir físicamente al aula. Aunque otorgando un significado más amplio al término inclusión podemos observar diversas maneras de usar la robótica educativa como estrategia para ello. Así, por ejemplo, hay estudios que muestran como mediante el uso de robótica se pueden mitigar ciertas circunstancias que padece el alumnado a causa de algún tipo de discapacidad o dificultad de aprendizaje, como puede ser tratar problemas de lenguaje. Dado que, al minimizar el efecto de estos problemas, se favorece su inclusión educativa.

Por otra parte, también debemos señalar el uso de robótica educativa con alumnado con TEA, siendo uno de los usos que más se recogen en esta revisión sistemática, afirmando que gracias a ello se fomentan sus habilidades sociales, entre otras.

\section{2 ¿QUÉ BENEFICIOS SE OBTIENEN CON EL USO DE LA ROBÓTICA EN LA INCLUSIÓN EDUCATIVA?}

Los estudios muestran numerosos beneficios del uso de robótica para fomentar la inclusión educativa, los cuales están presentes en diversos sentidos.

Uno de los beneficios del uso de robótica educativa para la inclusión lo encontramos en el contexto de aula hospitalaria. Así, González-González et al. (2021) señalan que tras trabajar con el kit de robótica las emociones de este alumnado cambian favorablemente. Así, las emociones negativas y las neutras disminuyen y las positivas aumentan, observándose, también, una mejora de las emociones del alumnado gracias al uso de robótica en otros contextos (Estévez et al., 2021).

Varios autores, como Valadão et al. (2016), Roberts-Yates \& Silvera-Tawil (2019), Fachantidis et al. (2019), Kumazaki et al. (2021) y Vicente-Castro et al. (2017), señalan mejoras en las habilidades sociales de alumnado con TEA gracias al uso de robótica, lo cual beneficia su inclusión educativa, incluyendo mejora en la comunicación verbal y no verbal (Valadão et al., 2016). También se indica una mejora en su comportamiento, incluso cuando éste es desafiante (Fachantidis et al., 2019; Roberts-Yates, \& Silvera-Tawil, 2019: Vicente-Castro et al., 2017). Además, mejora la confianza en sí mismos (Kumazaki et al., 2021).

Por otra parte, el artículo de Wood et al. (2019) muestra que es posible obtener mejora en la toma de perspectiva visual de algunos alumnos con TEA.

En general, alumnado de diversas características mejoran diversos aspectos que facilitan su inclusión educativa gracias al uso de robótica, como el trabajo en equipo (Bargagna et al., 2018; Lamptey et al., 2019), el aumento de la motivación y el interés por la tarea (Bargagna et al., 2018; Estévez et al., 2021; Lindsay et al., 2019; Roberts-Yates, \& Silvera-Tawil, 2019), mejora de la atención (Bargagna et al., 2018; Robles-Bykbaev et al., 2016; VelásquezAngamarca et al., 2019; Vicente-Castro et al., 2017), aumento de concentración (VicenteCastro et al., 2017) mejora en el comportamiento e incluso disminución de la agresividad (Estévez et al., 2021; Vicente-Castro et al., 2017) y aumento de autoestima (Encarnação et al., 2016; Estévez et al., 2021; Vicente-Castro et al., 2017).

Por otra parte, el uso de robótica educativa se erige como una buena ayuda en la terapia del habla con niños con diversas discapacidades, mejorando su lenguaje y facilitando el conseguir objetivos educativos. Lo cual se confirma con autores como Estévez et al. (2021), Robles-Bykbaev et al. (2016) y Velásquez-Angamarca et al. (2019). Además, también mejoran aspectos no verbales de la comunicación, como muestran Jeon et al. (2014).

Vicente-Castro et al. (2017) y Roberts-Yates \& Silvera-Tawil (2019) muestran otros aspectos que también se ven beneficiados, como la motricidad fina y la coordinación óculo manual al ensamblar las piezas para crear el robot.

Conchinha et al. (2015) reflejan una mejora en la aceptación de la frustración porque el alumnado se hace consciente de que con el error aprende al tener que hacer frente al desafío de solucionar el problema, concluyendo los autores lo importante del uso de robótica educativa 
pues favorece la inclusión e igualdad, así como ayuda a la adquisición de conocimientos sobre distintos campos.

Tras analizar la segunda pregunta de la investigación tenemos como resultado una larga lista de beneficios para la inclusión educativa derivados del uso de robótica, de los cuales podemos destacar el fomento del desarrollo en habilidades sociales, tanto en alumnado con TEA como en otros alumnos. Al hilo de lo cual podemos señalar también el aumento de la autoestima, la mejora del interés por la tarea, la mejora en la capacidad de trabajo en equipo y las mejoras en el comportamiento. Además, se observan beneficios en la motricidad derivados de la manipulación.

\section{3 ¿CÓMO ES LA FORMACIÓN DE LOS DOCENTES EN RELACIÓN CON EL USO DE LA ROBÓTICA PARA LA INCLUSIÓN?}

De esta revisión sistemática podemos deducir que hay poca investigación acerca de la formación docente en robótica enfocada a la inclusión o, al menos, ha sido encontrado poco al respecto con la estrategia de búsqueda utilizada. No obstante, sí se han obtenido algunos datos al respecto, los cuales se exponen a continuación.

Diversos estudios, como el realizado por Conchinha \& Correia de Freitas (2015) y el de Álvarez (2019) reflejan que los docentes tienen miedo antes de formarse acerca de robótica, pero al trabajar e ir familiarizándose con ella este miedo va desapareciendo y ponen en valor los beneficios que puede tener su uso para su alumnado.

Weibel et al. (2020) y Lindsay et al. (2019) apuntan que los docentes no tienen clara la manera de proceder y cómo utilizar el robot. Además, Lindsay et al. (2019) señalan que los docentes manifestaron no ser conscientes del grado de apoyo que brindaban a los niños ni de cómo proceder exactamente. Por lo que, una vez más, vemos que la clave es la formación docente, pues ésta brinda seguridad y promueve que un mayor número de profesores la pongan en práctica. Además, pese a que algunos docentes consideran que la robótica es compleja, sí quieren utilizarla con su alumnado, como indica Agatolio et al. (2017).

De hecho, como señalan Roberts-Yates \& Silvera-Tawil (2019), el grado de éxito por parte del alumnado al trabajar la robótica depende de la tarea realizada por el docente, como apuntábamos en el marco teórico. Además, las actividades deben estar bien diseñadas para sacarles el máximo provecho (Kucuk, \& Sisman, 2018), por lo que la formación docente resulta fundamental (Pittí et al., 2014).

Los docentes ven beneficioso, e incluso necesario, el uso de robótica educativa para la inclusión, como reflejan Encarnação et al. (2016). Además, estos autores muestran que los propios docentes desean recibir formación al respecto para poder aplicarla, mostrando éstos una actitud positiva al respecto (Desideri et al., 2020) como adelantábamos en el marco teórico (Moriña, \& Carballo, 2018).

Conchinha et al. (2015) señalan que, aunque la tecnología ha evolucionado mucho, esto no se ha visto reflejado en los planes de estudio y que el docente debe usarla y poseer los conocimientos necesarios para ello, hecho que ya se reflejaba en el marco teórico (Orcos, \& Aris, 2019).

Por tanto, vemos aquí diversos aspectos de la formación docente en distintas vertientes, aunque la revisión sistemática ha dado como resultado muy pocos estudios que traten el tema de la formación docente en relación al uso de la robótica para la inclusión. No obstante, destaca la idea de que los docentes mejoran su visión sobre el uso de robótica para la inclusión educativa y aumentan sus deseos de ponerla en práctica una vez se familiarizan con ella y disminuyen sus temores. Además, aun cuando les parece complicado anteponen el beneficio de su uso para los alumnos y muestran la intención de querer ponerlo en práctica. Así, la mayoría de docentes cuando reciben formación sobre la robótica educativa en su uso para la inclusión, la encuentran una estrategia muy útil. 


\subsection{LIMITACIONES Y LÍNEAS DE INVESTIGACIÓN FUTURAS}

Respecto a las limitaciones del estudio cabe señalar que, pese a que tras varias pruebas se consideró que la estrategia de búsqueda era apropiada, tras realizar la revisión sistemática se observa que se debería haber buscado más términos concretos para dar con mayor cantidad de estudios al respecto. Sobre todo, destaca lo escueto de los resultados con relación a la formación docente. Además, quizá haber abordado el término inclusión en su significado más amplio haya hecho que el resultado sea muy general.

Por otra parte, el tema tratado no parece estar muy abordado en la literatura actual, por lo que como líneas de investigación futuras se sugiere la realización de más estudios que aborden el uso de robótica educativa para la inclusión educativa. Y en lo referente a otras posibles revisiones sistemáticas, convendría realizarlas de alguna temática más concreta, pues la literatura encontrada sugiere que hay mucha información sobre el uso de robótica con alumnado con TEA.

Por otra parte, esta revisión sistemática no ha contado con ningún estudio que trate el uso de robótica para la inclusión con relación a las altas capacidades o alumnado con talentos específicos, siendo esta otra posible línea de investigación futura más específica.

\section{ENLACES}

Enlace para ver el Excel creado durante el proceso de desarrollo de la revisión sistemática: https://bit.ly/3nGznT0

\section{RECONOCIMIENTOS}

El presente artículo se deriva de mí Trabajo Fin de Máster realizado para el Máster Oficial Interuniversitario en Tecnología Educativa: e-Learning y Gestión del Conocimiento. Por ello quiero manifestar mi agradecimiento a quiénes lo forman, especialmente, a mi tutor del TFM.

\section{REFERENCIAS BIBLIOGRÁFICAS}

Agatolio, F., Pivetti, M., Di Battista, S., Menegatti, E., \& Moro, M. (2017). A training course in educational robotics for learning support teachers. En D. Alimisis, M. Moro, \& E. Menegatti, (Eds), Educational Robotics in the Makers Era (pp. 43-57). Springer. https://doi.org/10.1007/978-3-319-55553-9 4

Ainscow, M. (2016). Diversity and equity: A global education challenge. New Zealand Journal of Educational Studies, 51(2). https://doi.org/10.1007/s40841-016-0056-x

Ángel-Díaz, C.M., Segredo, E., Arnay, R., \& León, C. (2020). Simulador de robótica educativa para la promoción del pensamiento computacional. RED. Revista de Educación a Distancia, 20(63). http://doi.org/10.6018/red.410191

Asmy, F.S., Roberge-Milanese, A., Rombaut, M.C., Smith, G., \& Fels, D.I. (2020). WebMoti [Conferencia]. International Conference on Computers Helping People with Special Needs, Lecco, Italia. https://doi.org/10.1007/978-3-030-58805-2 18

Avello-Martínez, R., Lavonem, J., \& Zapata-Ros, M. (2020). Coding and educational robotics and their relationship with computational and creative thinking. A compressive review. RED: Revista Educación a Distancia, 62. https://doi.org/10.6018/red.413021

Azorín, C.M. (2017). Análisis de instrumentos sobre educación inclusiva y atención a la diversidad. Revista Complutense de Educación, 28(4), 1043-1060. https://doi.org/10.5209/RCED.51343

Bae, I., \& Han, J. (2017). Does height affect the strictness of robot assisted teacher? [Conferencia en papel]. IEEE International Conference on Human-Robot Interaction, Viena, Austria. https://doi.org/10.1145/3029798.3038401 
Bargagna, S., Castro, E., Cecchi, F., Cioni, G., Dario, P., Dell'Omo, M., Di Lieto, M.C., Inguaggiato, E., Martinelli, A., Pecini, C., \& Sgandurra, G. (2018). Educational robotics in Down Syndrome: A feasibility study. Technology, Knowledge and Learning, 24(2), 315-323. https://doi.org/10.1007/s10758-018-9366-z

Barrios-Aranibar, D., Gurgel, V., Santos, M., Araújo, G.R., Roza, V.C., Nascimento, R.A., Silva, A.F., Silvat, A.R.S., \& Gonçalves, L.M.G. (2006). RoboEduc: A software for teaching robotics to technological excluded children using Lego prototypes [Conferencia]. 2006 IEEE 3rd Latin American Robotics Symposium, Santiago, Chile. https://doi.org/10.1109/LARS.2006.334332

Basogain, X., \& Olmedo, M.E. (2020). Integración de pensamiento computacional en educación básica. Dos experiencias pedagógicas de aprendizaje colaborativo online. RED: Revista de Educación a Distancia, 20(63). http://doi.org/10.6018/red.409481

CASP. (2020). CASP Checklist. https://bit.ly/3yrKrW6

Conchinha, C., \& Correia de Freitas, J. (2015). Robots \& NEE: Learning by playing with robots in an inclusive school setting [Conferencia]. 2015 International Symposium on Computers in Education (SIIE), Setubal, Portugal. https://doi.org/10.1109/SIIE.2015.7451654

Conchinha, C., Osorio, P., \& Correia de Freitas, J. (2015). Playfulleaming: Educational robotics applied to students with learning disabilities [Conferencia en papel]. 2015 International Symposium on Computers in Education (SIIE), Setubal, Portugal. https://doi.org/10.1109/SIIE.2015.7451669

Desideri, L., Cesario, L., Gherardini, A., Fiordelmondo, V., Morganti, A., Malavasil, M., \& Hoogerwerf, E.J. (2020). Using a humanoid robot to promote inclusion of children with Autism Spectrum Disorders in mainstream classrooms: An overview of the RoBò service delivery approach. Life Span and Disability, 23(1), 41-51. https://bit.ly/2Y9egyi

Echeita, G. (2017). Educación inclusiva. Sonrisas y lágrimas. Aula Abierta, (46), 17-24. https://doi.org/10.17811/rifie.46.2017.17-24

Echeita, G., \& Ainscow, M. (2011). La educación inclusiva como derecho. Marco de referencia y pautas de acción para el desarrollo de una revolución pendiente. Tejuelo: Revista de Didáctica de la Lengua y la Literatura, (12), 26-46. https://bit.ly/3sX9hvl

Encarnação, P., Leite, T., Nunes, C., Nunes da Ponte, M., Adams, K., Cook, A., Caiado, A., Pereira, J., Piedade, G., \& Ribeiro, M. (2016). Using assistive robots to promote inclusive education. Disability and Rehabilitation: Assistive Technology, 12(4), 352-372. https://doi.org/10.3109/17483107.2016.1167970

Estévez, D., Terrón-López, M.J., Velasco-Quintana, P.J., Rodríguez-Jiménez, R.M., \& ÁlvarezManzano, V. (2021). A case study of a robot-assisted speech therapy for children with Language Disorders. Sustainability, 13(5), 2771. https://doi.org/10.3390/su13052771

Fachantidis, N., Syriopoulou-Delli, C.K., Vezyrtzis, I., \& Zygopoulou, M. (2019). Beneficial effects of a robot-mediated class activities on a child with ASD and his typical classmates. International Journal of Developmental Disabilities, 66(3), 245-253. https://doi.org/10.1080/20473869.2019.1565725

Fitter, N.T., Chowdhury, Y., Cha, E., Takayama, L., \& Mataric, M.J. (2018). Evaluating the effects of personalized appearance on telepresence robots for education [Conferencia]. IEEE International Conference on Human Robot Interaction, Chicago, IL, Estados Unidos. https://doi.org/10.1145/3173386.3177030

García, I., Romero, S., Escalante, L., \& Flores, V.J. (2018). Algunas propiedades psicométricas de las guías para evaluar prácticas inclusivas en el aula. REOP: Revista Española de $\begin{array}{lllll}\text { Orientación } y & \text { Psicopedagogía, } & \text { 8-28(2), }\end{array}$ https://doi.org/10.5944/reop.vol.29.num.2.2018.23150

González-González, C.S., Violant Holz, V., Infante-Moro, A., Cáceres-García, L., \& GuzmánFranco, M.D. (2021). Robótica educativa en contextos inclusivos: El caso de las aulas hospitalarias. Educación XXI, 24(1), 375-403. https://doi.org/10.5944/educXX1.27047 
Jadhav, D., Shah, P., \& Shah, H. (2018). A study to design VI classrooms using virtual reality aided telepresence [Conferencia]. International Conference on Advanced Learning Technologies, Mumbai, India. https://doi.org/10.1109/ICALT.2018.00080

Jeon, K.H., Yeon, S.J., Kim, Y.T., Song, S., \& Kim, J. (2014). Robot-based augmentative and alternative communication for nonverbal children with communication disorders [Conferencia]. UBICOMP 2014, Seattle, WA, Estados Unidos. https://doi.org/10.1145/2632048.2636078

Kärnä-Lin, E., Pihlainen-Bednarik, K., Sutinen, E., \& Virnes, M. (2007). Technology in Finnish special education - Toward inclusion and harmonized school days. Informatics in Education, 6(1), 103-104. https://bit.ly/3DDOdj4

Kucuk, S., \& Sisman, B. (2018). Pre-service teachers' experiences in learning robotics design and programming. Informatics in Education, 17(2), 301-320. https://doi.org/10.15388/infedu.2018.16

Kumazaki, H., Muramatsu, T., Yoshikawa, Y., Haraguchi, H., Sono, T., Matsumoto, Y., Ishiguro, H., Kikuchi, M., Sumiyoshi, T., \& Mimura, M. (2021). Enhancing communication skills of individuals with Autism Spectrum Disorders while maintaining social distancing using two tele-operated robots. Frontiers in Psychiatry, 11(598688). https://doi.org/10.3389/fpsyt.2020.598688

Lamptey, D.L., Cagliostro, E., Srikanthan, D., Hong, S., Dief, S., \& Lindsay, S. (2019). Assessing the impact of an adapted robotics programme on interest in Science, Technology, Engineering and Mathematics (STEM) among children with disabilities. International Journal of Disability, Development and Education, 68(1), 62-77. https://doi.org/10.1080/1034912X.2019.1650902

Lindsay, S., \& Hounsell, K.G. (2016). Adapting a robotics program to enhance participation and interest in STEM among children with disabilities: A pilot study. Disability and Rehabilitation: Assistive Technology, 12(7), 694-704. https://doi.org/10.1080/17483107.2016.1229047

Lindsay, S., Kolne, K., Oh, A., \& Cagliostro, E. (2019). Children with disabilities engaging in STEM: Exploring how a group-based robotics program influences STEM activation. Canadian Journal of Science, Mathematics and Technology Education, (19), 387-397. https://doi.org/10.1007/s42330-019-00061-x

Maestre, M.M., Nail, O., \& Rodríguez-Hidalgo, A.J. (2017). Desarrollo de competencias TIC y para la educación inclusiva en la formación inicial práctica del profesorado. Bordón: Revista de Pedagogía, 69(3), 57-72. https://doi.org/10.13042/Bordon.2017.51110

Martínez, L.G., Marrufo, S., Licea, G., Reyes-Juárez, J., \& Aguilar, L. (2018). Using a mobile platform for teaching and learning object oriented programming. IEEE Latin America Transactions, 16(6), 1825-1830. https://doi.org/10.1109/TLA.2018.8444405

Miranda, M., Burguera, J.L., Arias, J.M., \& Peña, E. (2018). Percepción del profesorado de orientación educativa de la atención a la diversidad en centros de primaria y secundaria en Asturias (España). REOP: Revista Española de Orientación y Psicopedagogía, 29(2), 71-86. https://doi.org/10.5944/reop.vol.29.num.2.2018.23154

Miranda-Pinto, M.S. (2019). Programación y robótica en Educación Infantil: Estudio multi caso en Portugal. Prisma Social, (25), 248-276. https://bit.ly/3zrZCjp

Mondada, F., Bonnet, E., Davrajh, S., Johal, W., \& Stopforth, R. (2016). R2T2: Robotics to integrate educational efforts in South Africa and Europe. International Journal of Advanced Robotic Systems, 13(5), 1-13. https://doi.org/10.1177/1729881416658165

Moriña, A., \& Carballo, R. (2018). Profesorado universitario y educación inclusiva: Respondiendo a sus necesidades de formación. Psicología Escolar e Educacional, 22, 8795. https://doi.org/10.1590/2175-3539/2018/053

Orcos, L., \& Aris, N. (2019). Percepciones del profesorado de Educación Secundaria ante la robótica educativa como recurso didáctico en el enfoque STEM. Opción, 35(90), 810-843. https://bit.ly/3yrozKC 
Pérez, E., Gilabert, A., Lorenzo, G., Lorenzo, A., \& Lledó, A. (2019). Educational intervention for students with asd: Emotion production and recognition through the Bee-Bot robot [Conferencia]. 11th International Conference on Education and New Learning Technologies, Palma, Mallorca, España. https://doi.org/10.21125/edulearn.2019.0972

Pittí, K., Curto, B., Moreno, V., \& Rodríguez, M.J. (2014). Uso de la robótica como herramienta de aprendizaje en Iberoamérica y España. VAEP-RITA, 2(1), 41-48. https://bit.ly/2XZgD6y

Quiroga, L.P. (2018). La robótica: Otra forma de aprender. Revista de Educación y Pensamiento, 25, 51-64. https://bit.ly/3sVY0fi

Roberts-Yates, C., \& Silvera-Tawil, D. (2019). Better education opportunities for students with autism and intellectual disabilities through digital technology. International Journal of Special Education, 34(1), 197-210. https://bit.ly/3Bm03w1

Robles-Bykbaev, V., Ochoa-Guaraca, M., Carpio-Moreta, M., Pulla-Sánchez, D., SerpaAndrade, L., López-Nores, M., \& García-Duque, J. (2016). Robotic assistant for support in speech therapy for children with cerebral palsy [Conferencia]. 2016 IEEE International Autumn Meeting on Power, Electronics and Computing (ROPEC 2016), Ixtapa, México. https://doi.org/10.1109/ROPEC.2016.7830603

Sánchez, J.L., \& Juárez, C. (2017). Modelo de robótica educativa con el robot Darwin Mini para desarrollar competencias en estudiantes de licenciatura. RIDE: Revista Iberoamericana para la Investigación y el Desarrollo Educativo, 8(15). https://doi.org/10.23913/ride.v8i15.325

Seo, J.Y., \& Richard, G.T. (2020). Coding through touch: Exploring and re-designing tactile making activities with learners with visual dis/abilities [Conferencia]. International Conference of the Learning Sciences, Nashville, Tennessee, Estados Unidos. https://bit.ly/3jip9Pu

Shute, V.J., Sun, C., \& Asbell-Clarke, J. (2017). Demystifying computational thinking. Educational Research Review, 22, 142-158. https://doi.org/10.1016/i.edurev.2017.09.003

Torturella-Valadão, C., Goulart, C., Rivera, H., Caldeira, E., Bastos-Filho, T.F., Frizera-Neto, A., \& Carelli, R. (2016). Analysis of the use of a robot to improve social skills in children with Autism Spectrum Disorder. Research on Biomedical Engineering, 32(2), 161-175. https://doi.org/10.1590/2446-4740.01316

Urrútia, G., \& Bonfill, X. (2010). Declaración PRISMA: Una propuesta para mejorar la publicación de revisiones sistemáticas y metaanálisis. Medicina clínica, 135(11), 507-511. https://doi.org/10.1016/j.medcli.2010.01.015

Velásquez-Angamarca, V., Mosquera-Cordero, K., Robles-Bykbaev, V., León-Pesántez, A., Krupke, D., Knox, J., Torres-Segarra, V., \& Chicaiza-Juela, P. (2019). An educational robotic assistant for supporting therapy sessions of children with communication disorders [Conferencia]. 2019 7th International Engineering, Sciences and Technology Conference (IESTEC), Panamá, Panamá. https://doi.org/10.1109/IESTEC46403.2019.00110

Vicente-Castro, F., Maldonado-Briegas, J.J., González-Ballester, S., \& Vera-González, D. (2017). Actividad extraescolar para aprender a aprender: La robótica como herramienta educativa. Revista de Estudios e Investigación en Psicología y Educación, (13), 124-128. https://doi.org/10.17979/reipe.2017.0.13.2542

Weibel, M., Nielsen, M.K.F., Topperzer, M.K., Hammer, N.M., Møller, S.W., Schmiegelow, K., \& Larsen, H.B. (2020). Back to school with telepresence robot technology: A qualitative pilot study about how telepresence robots help school-aged children and adolescents with cancer to remain socially and academically connected with their school classes during treatment. Nursing Open, 7(4), 988-997. https://doi.org/10.1002/nop2.471

Wing, J.M. (2006). Computational Thinking: It represents a universally applicable attitude and skill set everyone, not just computer scientists, would be eager to learn and use. Communications of the ACM, 49(3), 33-35. https://bit.ly/3zxKJfk

Wood, L.J., Robins, B., Lakatos, G., Syrdal, D.S., Zaraki, A., \& Dautenhahn, K. (2019). Developing a protocol and experimental setup for using a humanoid robot to assist children 
with autism to develop visual perspective taking skills. Paladyn, Journal of Behavioral Robotics, (10), 167-179. https://doi.org/10.1515/pibr-2019-0013

Zamin, N., Arshad, N.I., Rafiey, N., \& Hashim, A.S. (2018). Robotic teaching aid for disabled children: A sustainable solution for Industrial Revolution 4.0. International Journal of Engineering \& Technology, 7(2.28), 200-203. https://doi.org/10.14419/ijet.v7i2.28.12912

Zapata-Ros, M. (2015). Pensamiento computacional: Una nueva alfabetización digital. RED: Revista de Educación a Distancia, (46), 1-47. https://bit.ly/3zvFFbB

Zhang, G., \& Hansen, J.P. (2020). People with motor disabilities using gaze to control telerobots [Conferencia]. CHI 2020 Late-Breaking Work, Honolulu, HI, Estados Unidos. https://doi.org/10.1145/3334480.3382939

\section{INFORMACIÓN SOBRE LA AUTORA}

\section{Judit Rodrigo Parra}

Universidad de Murcia

Graduada como Técnico Superior en Educación Infantil y también, maestra de Pedagogía Terapéutica. Máster Oficial Interuniversitario en Tecnología Educativa: e-Learning y Gestión del Conocimiento. Las principales líneas de investigación son aquellas relacionadas con la tecnología educativa, sobre todo la robótica, el pensamiento computacional y la programación. Por otra parte, también las relacionadas con la educación especial.

Los textos publicados en esta revista están sujetos a una licencia de Reconocimiento 4.0 España de Creative Commons. Puede copiarlos, distribuirlos, comunicarlos públicamente y hacer obras derivadas siempre que reconozca los créditos de las obras (autoría, nombre de la revista, institución editora) de la manera especificada por los autores o por la revista. La licencia completa se puede consultar en:Licencia Creative Commons Atribución-NoComercial-Compartir por igual 4.0 Internacional. 